\title{
Organizing, Professionalisierung, Vernetzung
}

\section{Aktuelle Entwicklungen der wohnungspolitischen Bewegung in Berlin, Hamburg, Jena und Leipzig}

\author{
Lina Hurlin • Elodie Vittu • Anne Vogelpohl (D) - Lisa Vollmer • \\ Marcel Weikert
}

Eingegangen: 22. Juni 2021 / Angenommen: 12. Oktober 2021 / Online publiziert: 18. November 2021 (C) The Author(s), under exclusive licence to Springer Fachmedien Wiesbaden GmbH, ein Teil von Springer Nature 2021

Zusammenfassung Seit vielen Jahren gibt es in Deutschland eine kraftvolle wohnungspolitische Bewegung. Sie kritisiert steigende Mieten, Gentrifizierung, mangelnde Mitbestimmungsmöglichkeiten oder die Unterversorgung von Wohnungslosen und Geflüchteten. Zugleich organisiert sie Mieter:innen, alternative Wohnraumpolitiken und Kampagnen. Anhand von Einblicken aus Berlin, Hamburg, Jena und Leipzig stellen wir die neuesten Entwicklungen der Bewegung heraus. Im Austausch über die vier Städte haben wir drei jüngere Tendenzen im kollektiven Protest ausgemacht: eine vermehrte Anwendung des Community Organizing, eine Institutionalisierung und Professionalisierung sowie eine intensivierte Vernetzung. Darüber hinaus haben wir zwei Spannungsfelder identifiziert, die innerhalb der Bewegung kontrovers diskutiert werden: der Umgang mit alten und neuen Partizipationsangeboten sowie deren potenzielle Folgen für eine Vereinnahmung der Bewegung; und die Debatte über die ,richtige“ Strategie zur Erreichung der Fernziele Dekommo-

Lina Hurlin, M.Sc. Urbanistik

Leipzig, Deutschland

E-Mail: lina.hurlin@posteo.de

Elodie Vittu · Lisa Vollmer

Institut für Europäische Urbanistik (IfEU), Bauhaus-Universität Weimar, Weimar, Deutschland

Elodie Vittu

E-Mail: elodie.vittu@uni-weimar.de

Lisa Vollmer

E-Mail: lisa.vollmer@uni-weimar.de

Anne Vogelpohl $(\bowtie)$

Department Soziale Arbeit, HAW Hamburg, Hamburg, Deutschland

E-Mail: anne.vogelpohl@haw-hamburg.de

Marcel Weikert

Masterstudiengang Urbanistik, Bauhaus-Universität Weimar, Weimar, Deutschland

E-Mail: marcel.weikert@uni-weimar.de 
difizierung und Vergesellschaftung der Wohnraumversorgung. Ein Ausblick zeigt anstehende Herausforderungen auf, die sich auch durch die Pandemie dringlicher und teilweise neu stellen.

Schlüsselwörter Stadtpolitik · Mieten · Soziale Bewegungen · Verdrängung · Dekommodifizierung

\title{
Organizing, professionalization, networking
}

Current developments in the housing movement in Berlin, Hamburg, Jena and Leipzig

\begin{abstract}
For many years, there has been a powerful housing movement in Germany. It criticizes housing issues like rising rents, gentrification, lack of participatory opportunities or the lack of basic provision for homeless people and refugees. Likewise, this social movement supports tenants and alternative housing policies and organizes campaigns. Drawing on insights from Berlin, Hamburg, Jena and Leipzig, we highlight the latest developments of the housing movement in this paper. By describing the movement in these four cities, we have found three recent trends in collective protest: an increased use of the approach "community organizing", an overall institutionalization and professionalization as well as an intensified networking. Moreover, we have identified two areas which are being controversially discussed within the movement: how to deal with old and new participatory opportunities and their potential challenges and consequences for appropriating the movement; and the debate over the "right" strategy for achieving the core goals of decommodification and communization of housing provision. In concluding remarks we raise additional challenges that have become, particularly in light of the COVID19 pandemic, even more pressing.
\end{abstract}

Keywords Urban policy · Rents · Social movements · Gentrification · Decommodification

„Wohnraum darf keine Ware sein“ - so lautet die gängige Antwort auf die Wohnungsfrage (Holm 2014; Schönig et al. 2017) seitens der wohnungspolitischen Bewegung Deutschlands. Wie hat sich diese Bewegung in den letzten Jahren entwickelt? Welche Strategien verfolgt sie? Diese Fragen untersuchen wir in diesem Beitrag anhand der wohnungspolitischen sozialen Bewegung in Jena, Leipzig, Hamburg und Berlin.

Wenn es einen gemeinsamen Nenner innerhalb der Bewegung gibt, dann dürfte sich dieser auf die Fernziele einer Dekommodifizierung und Vergesellschaftung der Wohnraumversorgung belaufen. Mit diesem utopischen Überschuss versuchen im letzten Jahrzehnt zahlreiche Akteur:innen lokal, regional und bundesweit eine organisierte wohnungspolitische Bewegung aufzubauen. Es ist ihr Verdienst, dass Alternativen zur neoliberalen Wohnungspolitik sichtbar und teilweise mehrheitsfähig wurden (Vogelpohl und Buchholz 2017; Vollmer und Kadi 2018). 
Anknüpfend an diese „Repolitisierung des Wohnens“ (Vogelpohl et al. 2017), wird hier die Entwicklung der Bewegung in Jena, Leipzig, Berlin und Hamburg in den letzten vier Jahren beschrieben. Der Beitrag baut dabei auf einen Artikel auf, in dem die Erfolge, aber auch Gegenkräfte und Herausforderungen der wohnungspolitischen Bewegung in den vier unterschiedlichen Städten vergleichend dargestellt wurden (ebd.). Die Kritik der Bewegung an steigenden Mieten, Gentrifizierungsprozessen und Verdrängung, mangelnden Mitbestimmungsmöglichkeiten, der Unterversorgung von Wohnungslosen oder Geflüchteten sowie ihre Arbeit an alternativen Politiken und Konzepten der Wohnraumversorgung hat sich in den vergangenen vier Jahren nicht wesentlich verändert. Es sind allerdings einige neue Tendenzen in ihrer strategischen Ausrichtung zu beobachten, und spannungsvolle Debatten haben sich entlang verschiedener Linien deutlicher herauskristallisiert ${ }^{1}$.

Um dies zu analysieren, beschreiben wir zunächst kurz die Entwicklung der Bewegung sowie die wohnungspolitischen Entwicklungen in den vier Städten in Form von Steckbriefen. Anschließend stellen wir drei in allen vier Städten zu beobachtende Tendenzen vor: (1) Die wohnungspolitische Bewegung nimmt zunehmend Bezug auf Ansätze des Community Organizing; (2) einzelne Bewegungsakteure institutionalisieren und professionalisieren sich stärker; (3) die Vernetzung innerhalb der wohnungspolitischen Bewegung sowie die Kooperation mit anderen zivilgesellschaftlichen Akteuren jenseits sozialer Bewegungen nimmt zu. Neben diesen allgemeinen Tendenzen lassen sich zwei Spannungsfelder identifizieren, die innerhalb der Bewegung kontrovers diskutiert werden: (1) Die Bewegung ist mit der Frage konfrontiert, wie sie mit alten und neuen Partizipationsangeboten, der Gefahr der Vereinnahmung und den Herausforderungen von selbstermächtigenden Prozessen umgeht; (2) außerdem werden in der Bewegung kontroverse Debatten über Organisations- und Aktionsformen und die ,richtige“ Strategie zur Erreichung der Ziele - der Dekommodifizierung und Vergesellschaftung der Wohnraumversorgung - geführt. Das Fazit gibt einen Ausblick auf weitere Herausforderungen, vor der die Bewegung steht und die durch die Pandemie umso dringlicher geworden sind.

\section{Die wohnungspolitische Bewegung in den vier Fallbeispielen}

Berlin, Hamburg, Jena und Leipzig ${ }^{2}$ unterscheiden sich in ihrer Größe sowie der Struktur ihrer Wohnraumversorgung. Während in Leipzig und Jena im Umgang mit der Wohnungskrise kommunale Instrumente zur Verfügung stehen, können Hamburg und Berlin als Stadtstaaten auf weitergehende Regelungskompetenzen zurückgrei-

\footnotetext{
1 Alle Autor:innen agieren sowohl forschend als auch aktiv in der wohnungspolitischen Bewegung in den hier diskutierten Städten. Schwerpunkt ihres Aktivismus und ihrer Forschung hat Lisa Vollmer in Berlin, Anne Vogelpohl in Hamburg, Élodie Vittu und Marcel Weikert in Jena und Lina Hurlin in Leipzig. Jahrelange teilnehmende Beobachtungen, zahlreiche Interviews und Sekundärdatenanalysen, teils im Rahmen von Abschlussarbeiten und Promotionen erstellt, gehen in diesen Beitrag ein. Die vergleichende Betrachtung der vier Städte beruht auf einem laufenden Austausch der Autor:innen, vor und während des Schreibprozesses dieses Aufsatzes.

2 Wir führen die Städte in alphabetischer Reihenfolge auf; eine inhaltliche Gewichtung ist damit nicht verbunden.
} 
Tab. 1 Entwicklungen auf dem Wohnungsmarkt in Berlin, Hamburg, Jena und Leipzig

\begin{tabular}{|c|c|c|c|c|}
\hline & Berlin & Hamburg & Jena & Leipzig \\
\hline Einwohner:innenzahl (2021) & 3.748 .148 & 1.904 .444 & 108.940 & 605.203 \\
\hline Mietquote (2019) & $85 \%$ & $77 \%$ & $79 \%$ & $88 \%$ \\
\hline $\begin{array}{l}\text { \%-Anteil kommunale/öffentliche Wohnun- } \\
\text { gen am Gesamtwohnungsbestand }\end{array}$ & $\begin{array}{l}15,5 \% \\
(2019)\end{array}$ & $\begin{array}{l}14 \% \\
(2020)\end{array}$ & $\begin{array}{l}23,7 \% \\
(2019)\end{array}$ & $\begin{array}{l}12 \% \\
(2019)\end{array}$ \\
\hline $\begin{array}{l}\text { \%-Anteil genossenschaftliche Wohnungen } \\
\text { am Gesamtwohnungsbestand }\end{array}$ & $\begin{array}{l}10 \% \\
(2019)\end{array}$ & $\begin{array}{l}14,2 \\
(2020)\end{array}$ & $\begin{array}{l}27 \% \%^{\mathrm{a}} \\
(2019)\end{array}$ & $\begin{array}{l}16,5 \% \\
(2019)\end{array}$ \\
\hline $\begin{array}{l}\text { \%-Anteil geförderter Wohnraum (sozialer } \\
\text { Wohnungsbau) am Gesamtwohnungsbe- } \\
\text { stand (2019) }\end{array}$ & $4,9 \%$ & $8,2 \%$ & $1,2 \%$ & $0,1 \%$ \\
\hline $\begin{array}{l}\text { Ortsübliche Vergleichsmiete (nettokalt), } \\
2020\end{array}$ & $7,13 €$ & $8,60 €$ & $7,11 €$ & $5,33 €$ \\
\hline $\begin{array}{l}\text { Mietsteigerung (nettokalt) der ortsüblichen } \\
\text { Vergleichsmiete } \\
(2005-2020)\end{array}$ & $92 \%$ & $55 \%$ & $34 \%$ & $44 \%$ \\
\hline $\begin{array}{l}\text { Durchschnittliche Angebotsmiete (netto- } \\
\text { kalt) }\end{array}$ & $\begin{array}{l}10,68 € / q m \\
(2019)\end{array}$ & $\begin{array}{l}11,46 € / q m \\
(2021)\end{array}$ & $\begin{array}{l}9,32 € / q m \\
(2018)\end{array}$ & $\begin{array}{l}7,00 € / q m \\
(2019)\end{array}$ \\
\hline $\begin{array}{l}\text { Verfügbares Einkommen der privaten } \\
\text { Haushalte je Einwohner:in, } 2018\end{array}$ & $20.972 €$ & $25.029 €$ & $18.919 €$ & $18.825 €$ \\
\hline
\end{tabular}

Quellen: Amt für Statistik und Wahlen Leipzig (2021), Bürgerschaft der FHH (2020), empirica regio (2021), F+B (2021), Statistische Ämter des Bundes und der Länder (2021), IBB (2020), Stadt Jena (2020) a Geschätzt

fen. Das gilt auch umgekehrt für soziale Bewegungen, die hier Einfluss auf zwei politische Ebenen gewinnen können und darüber hinaus z. B. durch Volksentscheide auf Landesebene auch weitere demokratische Mittel zur Verfügung haben. Auch ist die Wohnungsversorgung in den Städten verschieden strukturiert (Tab. 1). Ungeachtet dieser Unterschiede ist es in allen vier Städten im vergangenen Jahrzehnt zu signifikanten Mietsteigerungen gekommen, die zusammen mit unzulänglichen Partizipationsverfahren, Diskriminierung auf dem Wohnungsmarkt und Verdrängungsprozessen die Ausgangslage der wohnungspolitischen Proteste in den Städten bilden.

\subsection{Steckbrief Berlin}

In Berlin hat sich in Sachen Wohnungspolitik in den letzten Jahren und seit unserem letzten Bericht aus den vier Städten (Vogelpohl et al. 2017) einiges getan. Die institutionelle Politik hat mit zahlreichen Maßnahmen auf die sich zuspitzende Wohnungsfrage reagiert. Der Wohnungsmarkt wurde durch zahlreiche soziale Erhaltungsgebiete, eine Zweckentfremdungsverbotsverordnung und andere lokale Maßnahmen stärker reguliert. Flächendeckend hat dagegen der Mietendeckel in der kurzen Zeit seines Bestehens verdeutlicht, was möglich wäre: Nicht nur eine Dämpfung der Mietpreisspirale, sondern ein Absenken des gesamten Mietniveaus hätte Berliner Mieter:innen entlastet, hätte das Bundesverfassungsgericht nicht entschieden, dass eine solche Regelungskompetenz nicht in der Hand eines Bundeslandes liegt. Auch im Bereich des Neubaus hat die Berliner Regierung auf die Wohnungsfrage reagiert, vor allem durch die landeseigenen Wohnungsunternehmen. Das öffentliche Wohnungsmarktsegment wird darüber hinaus durch Ankäufe, teils über 
das Vorkaufsrecht, ausgeweitet. Die landeseigenen Wohnungsunternehmen werden außerdem nicht mehr als „,cash cows“ für die Haushaltssanierung benutzt, sondern wieder für soziale wohnungspolitische Ziele eingesetzt. Die Quote von preis- und belegungsgebundenen Wohnungen im landeseigenen Bestand und Neubauprojekten ist kontinuierlich gestiegen, auf derzeit angestrebte $63 \%$ bzw. $60 \%$ (Vollmer und Kadi 2018; Holm 2020; Kadi et al. 2021).

Möglich wurden diese und andere Politiken einer Abkehr von neoliberaler Wohnungspolitik durch den kontinuierlichen Druck der wohnungspolitischen Bewegung (Vollmer 2018, 2019b). Entgegen mancher Prophezeiung wurde die Bewegung von der rot-rot-grünen Regierungskoalition seit 2016 nicht eingehegt oder durch deren Maßnahmen beruhigt. Der Bewegung gelingt es weiterhin, das Thema in der lokalen Öffentlichkeit äußerst präsent zu halten. Wie schon in den Jahren davor, gründen sich dauernd neue Initiativen, meist aus Betroffenheit in einem Haus oder einer Siedlung. Die stadtweite Vernetzung der wohnungspolitischen Initiativen ist dabei prekär geblieben und konzentriert sich hauptsächlich auf die Organisation der jährlichen „Mietenwahnsinn“-Demonstration. Die Trägerschaft und Strategien der Bewegung sind sehr heterogen - von der durch Rentner:innen besetzen räumungsbedrohten Senior:innenfreizeiteinrichtung bis zur unterschriftensammelnden linksradikalen Gruppe.

Es ist insgesamt eine zunehmende Dynamik der Bewegung zu beobachten, gespeist aus bisherigen Erfolgen und gestiegenen realen Handlungsmöglichkeiten. Zwei Beispiele illustrieren dies: Die Ausübung des Vorkaufsrechts (Sarnow 2019) in Milieuschutzgebieten wurde von der Bewegung erkämpft. Dies hat umgekehrt zur Gründung zahlreicher Hausinitiativen geführt, die fordern, in die öffentliche Hand überführt zu werden. Die Grenzen des Instruments Vorkaufsrecht, insbesondere durch seine hohen Kosten, werden gleichzeitig von der Bewegung problematisiert.

Ein weiteres Beispiel für die Zuspitzung der Bewegung ist die Kampagne „Deutsche Wohnen \& Co enteignen“ (Abb. 1). Sie strebt nach dem Volksentscheid zum Tempelhofer Feld und dem Mietenvolksentscheid bereits das dritte wohnungspolitische Referendum in Berlin an mit den Zielen, den Wohnungsbestand in die öffentliche Hand zu überführen und zu demokratisieren. Die Kampagne speist ihren Erfolg in Hinsicht auf Öffentlichkeitswirksamkeit und Mobilisierung teils aus die-

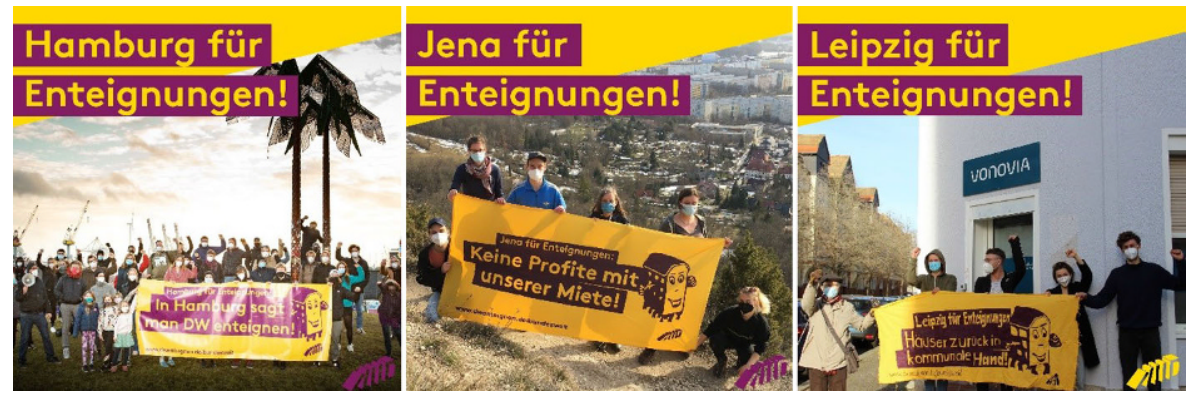

Abb. 1 Solidaritätsaktion bei der Kampagne „Deutsche Wohnen \& Co enteignen“: Solidaritätsbekundungen aus Hamburg, Jena und Leipzig. (@) dwenteignen) 
sen jahrelangen Routinen, teils aus der in den letzten Jahren eher neuen Erfahrung des Organizing, insbesondere in den Beständen der „Deutsche Wohnen“. Der als Beruhigungsmittel vom Senat erdachte Mietendeckel konnte die Dynamik der Enteignungskampagne nicht bremsen, im Gegenteil schien er eher den Horizont des Möglichen zu erweitern. Und nach dessen Aus im Frühjahr 2021 ließen 15.000 Demonstrierende auf einer morgens angekündigten Demo am Abend wissen: „Gegen den Mietenwahnsinn - Jetzt erst recht!“.

\subsection{Steckbrief Hamburg}

Abriss und Neubau - so lässt sich die Stadtentwicklungspolitik der letzten Jahrzehnte in Hamburg zusammenfassen. Und das prägt auch die wohnungspolitische Bewegung, die Alternativen dazu erkämpft. In den 1980er Jahren sollte durch Abriss Platz in der zentralen Stadt für Bürokomplexe geschaffen werden; das wurde u.a. durch die bekannten Proteste in der Hafenstraße verhindert. Das ,Recht auf Stadt“Netzwerk (RaS) als jüngste Formierung der Bewegung hat 2009 wichtige Impulse aus der Besetzung des zum Abriss freigegebenen Gängeviertels erhalten (vgl. zu der Entwicklung Rinn 2016).

Wohnungspolitisch ist in der Hansestadt „Neubau“ die Antwort des Hamburger Senats auf zu wenige und zu teure Wohnungen - teilweise auf Basis von Abriss, teilweise durch Nachverdichtung und Konversion. Im Bündnis mit der Wohnungswirtschaft werden zurzeit jährlich 10.000 neue Wohnungen genehmigt (Metzger 2020). Hamburg gilt unter Kommunalpolitiker:innen und in der Immobilienwirtschaft inzwischen als Musterstadt für eine effektive Neubaupolitik. Auch deshalb wird der Fokus auf Neubau in der Hamburger Regierung nicht hinterfragt; er scheint sowohl politische Mehrheiten zu sichern als auch Probleme auf dem Wohnungsmarkt zu lösen (Kemper und Vogelpohl 2020). Auf Neubau mit einem Drittelmix zu setzen, war 2011 tatsächlich innovativ. Der Bedarf an Neubau wurde damals wie heute auch von der wohnungspolitischen Bewegung kaum in Frage gestellt (Pott 2020). Nur sind seither nicht wesentlich neue Formate der Wohnungspolitik hinzugekommen. Zwar wird auch in Hamburg auf Soziale Erhaltungsverordnungen oder Konzeptvergabe gesetzt; aber die konsequente Nutzung von Vorkaufsrechten und des Erbbaurechts sowie die Kopplung dieser an Gemeinnützigkeit spielt keine größere Rolle (ebd.). Debatten um Enteignungen (besitzt die Stadt doch mit der SAGA bereits viele Wohnungen) oder um den Mietendeckel haben in Hamburg kaum Nährboden gefunden.

Bereits 2009 waren es soziale Bewegungen, die das Thema Wohnen auf die politische Agenda gebracht hatten. Damals gründete sich in Hamburg das deutschlandweit erste größere RaS-Netzwerk (vgl. zur Gründung Vittu 2021; Rinn 2016), in dem nicht nur Wohnen und Mieten thematisiert wird, sondern auch Partizipation, Ökologie, rassistische Diskriminierung und weitere Facetten im Kampf gegen eine neoliberalisierte Stadtentwicklungspolitik. RaS ist in Hamburg bis heute kein vorrangig wohnungspolitisches Netzwerk, sondern thematisiert ebenso Gewerbe, rassistische Ausgrenzung in Stadtquartieren (vor allem St. Pauli) oder (mangelnde) Partizipation. Wohnungspolitisch adressiert das Netzwerk die blinden Flecken des starken Neubaufokus: Neben Kritik an Spekulation betrifft dies vor allem politi- 


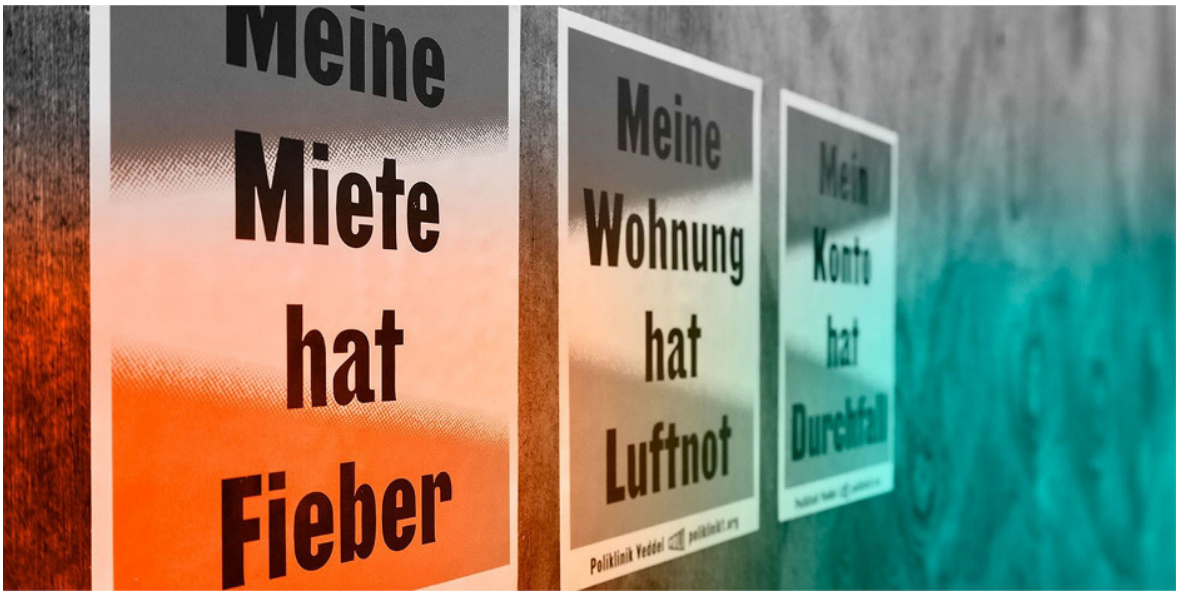

Abb. 2 Plakataktion der Poliklinik Veddel, die auf Wechselwirkungen zwischen der Wohnungsproblematik und Gesundheitsversorgung aufmerksam macht. (ㄷ Poliklinik Veddel)

sche Lösungen für den Bestand, in dem Mieten rasant gestiegen sind und in dem Überbelegung keine Seltenheit ist.

Umgekehrt besteht die wohnungspolitische Bewegung keineswegs nur aus dem RaS-Netzwerk und den darin vernetzten Initiativen. Die Poliklinik Veddel beispielsweise setzt an der Gesundheitsfrage an, verknüpft diese aber ebenfalls eng mit der Wohnungsfrage (Abb. 2).

Insgesamt setzt sich der wohnungspolitische Protest aus vier Formen zusammen, die in enger, sich positiv aufeinander beziehender Wechselwirkung stehen: Erstens gibt es eine Reihe von Einzelinitiativen, die sich gegen den Abriss oder Verkauf eines Wohn- bzw. Mischkomplexes wehren. Zweitens gibt es thematisch fokussierte Initiativen über Einzelfälle hinweg, die sich z. B. mit Problematiken der Obdachlosigkeit, der Unterbringung von Geflüchteten, der Überbelegung oder einzelner Vermietungsgesellschaften auseinandersetzen. Drittens gibt es nach wie vor die Vernetzung dieser Initiativen im RaS-Netzwerk. Und viertens gibt es seit 2019 Bemühungen um zwei Volksinitiativen unter dem Motto „Keine Profite mit Boden und Miete". Diese Initiativen sind stark getragen von den beiden Hamburger Mieter:innenvereinen und einzelnen Aktiven, gekoppelt an einen „Mietenpolitischen Ratschlag“, der mietenpolitische Aktivist:innen vernetzt.

\subsection{Steckbrief Jena}

„Die Schatten der Lichtstadt“ ist der wohnungspolitische Slogan der Recht auf StadtBewegung in der sogenannten „Saalemetropole“ Jena (Göbel 2020). Hier werden derzeit Baulücken geschlossen, die Stadt mit ihrer Tallage mit Mehrfamilienhäusern verdichtet, Plattenbauten modernisiert sowie neue Flächen für Einfamilienhäuser geschaffen. Obschon sich mehr als $50 \%$ des Wohnungsbestandes in kommunalem bzw. in genossenschaftlichem Eigentum befinden, steht Jena vor großen wohnungspolitischen Herausforderungen. Trotz erhöhter Neubautätigkeit sowie Einführung 




Abb. 3 2. Thüringer Mietparade 2019. (@ Recht auf Stadt Jena)

verschiedener Steuerungsinstrumente wie Mietpreisbremse, Kappungsgrenze, $20 \%$ Anteil von Sozialwohnungen im Geschosswohnungsbau und Konzeptvergabe (Jacobs 2020) stiegen die Angebots- und Bestandsmieten in den letzten Jahren weiter, wenn auch mit abnehmendem Tempo (TMIL 2019). Soziale Folgeprobleme wie eine hohe Mietbelastung für einkommensschwache Haushalte, starke sozialräumliche Polarisierungstendenzen und Versorgungsdefizite mit angemessenem und bezahlbarem Wohnraum für breite Schichten provozieren daher auch weiterhin Kritik innerhalb (Jacobs 2020) sowie außerhalb der Parlamente (Vogelpohl et al. 2017; Vollmer 2019a; Müller und Vollmer 2020; Abb. 3).

Die Ausreizung des kommunalen Spielraums gehört heute zu den Forderungen der wohnungspolitischen Bewegung. Darin zeigt sich ein strategischer Richtungswechsel. In den frühen 2010er Jahren standen noch Versuche im Vordergrund, einer abstrakten Kritik an der Kommodifizierung der Wohnraumversorgung Gehör zu verschaffen. Diese in der Regel ausschließlich von Studierenden getragenen Kampagnen blieben jedoch ebenso unsichtbar wie kurzlebig. Seit Mitte der 2010er Jahre entstanden dagegen verschiedene Initiativen, die die konkrete Verletzung von Mieter:inneninteressen zum Anlass für die Organisierung von Mieter:innen nahmen (Müller und Vollmer 2020). Diese aus dem losen Recht auf Stadt-Netzwerk hervorgegangenen Akteure arbeiten auf Grundlage direkter Betroffenheit, setzen so punktuelle Akzente und leisten kontinuierliche Vermittlungsarbeit an der Basis (Vittu 2021).

Der seit 2015 organisierte „Miettreff“ ist eine Unterstützungsstruktur für Betroffene von Mietproblemen. Und in Reaktion auf Mieterhöhungen von einzelnen Wohnungsunternehmen gründeten Mieter:innen der ,jenawohnen“ 2017 die „Bür- 
gerinitiative für soziales Wohnen in Jena“ (BI) sowie 2018 die „Initiative zur ReDemokratisierung der Jenaer Baugenossenschaft“ (JBG-Initiative). Als ideologische Klammer dient die Forderung nach Demokratisierung der Unternehmen. Denn die direkte Durchsetzung von Mieter:inneninteressen erscheint den Initiativen als sinnvoller Weg zum nicht aufgegebenen langfristigen Ziel einer Dekommodifizierung der Wohnraumversorgung. Die BI erkämpfte, dass drei Mieter:innen im Aufsichtsrat der ,jenawohnen“ vertreten sind. Als Treiber der großen Ziele Dekommodifizierung und Demokratisierung dienen kontinuierliche kleine Kämpfe. So organisierte die BI 2020 Mieter:innen, die von Verdrängung auf Grund von Sanierungsprojekten bedroht sowie von unzulässigen Nebenkostenabrechnungen betroffen waren.

Die beiden genannten Initiativen agieren räumlich in jenen Stadtteilen, in denen sich die Bestände der jeweiligen Wohnungsunternehmen konzentrieren; die JBGInitiative in Jena-Süd und die BI in Jena-Lobeda. Hier verschränken sich Mieter:innenorganisierung und Nachbarschaftsarbeit. Beide Initiativen geben mittlerweile eine eigene Stadtteil- bzw. Mieter:innenzeitung heraus. Mit der „Magdelstube“ entstand in Jena-Süd eine konkrete Anlaufstelle zum Kennenlernen, Austauschen und Organisieren. Nicht zufällig entstanden in diesen beiden Stadtteilen im März 2020 die größten Corona-Solidaritätsstrukturen. Im Winter 2021 beteiligten sich die Initiativen zudem federführend an einem stadtweiten Bündnis gegen kommunale Haushaltskürzungen.

\subsection{Steckbrief Leipzig}

Einige Jahre hat es gedauert, bis Leipzig das Narrativ der schrumpfenden Stadt und die damit einhergehende Aufwertungspolitik abschütteln konnte. Irgendwann war es dann in die tiefsten Ecken der Stadtverwaltung gedrungen: Leipzig wächst, und es muss etwas unternommen werden. Die Angst vor steigenden Mieten war plötzlich in aller Munde. Hohe Zahlen wurden prophezeit: ein Bevölkerungswachstum von bis zu 20.000 Menschen im Jahr. Ganz so ist es dann doch nicht gekommen. Für 2030 prognostizierte das Amt für Statistik und Wahlen (2016) noch eine Bevölkerungszahl von 721.800 Einwohner:innen. 2019 ist das Amt mit den Schätzungen schon deutlich zurückgerudert und hat nun - ebenfalls für 2030 - eine geschätzte Bevölkerungszahl von 644.300 angegeben (Amt für Statistik und Wahlen Leipzig 2019).

Nach wie vor sind Entmietungen und Verdrängung sowie spekulativer Leerstand Probleme, die Leipziger:innen umtreiben. Wohl nicht zuletzt als Reaktion auf Proteste, reagiert nun auch in Leipzig die Stadtpolitik. Neben der unermüdlichen Arbeit stadtpolitischer Gruppen (Netzwerk Leipzig-Stadt für Alle, Vernetzung Süd, Leipzig für alle) sind es auch Einzelmobilisierungen, wie zur „Mietenwahnsinn“-Demonstration im Jahr 2019 mit 3000 Teilnehmer:innen (Tappert 2019), die als Korrektiv auf Stadtverwaltung und -politik einwirken. Mit der Einführung von Milieuschutzgebieten zur Kontrolle von Modernisierung und der Ausübung von Vorkaufsrechten im Jahr 2020 geht die Kommune einen Schritt in Richtung Mieter:innenschutz.

Die stadtpolitische Bewegung ist in Leipzig so vielfältig wie in anderen Städten auch. Leipzig ist aber auch bekannt für seine Autonome Szene, die sich in den letzten Jahren ebenfalls vermehrt zum Thema Wohnungspolitik äußert. Im September 2020 wurden sowohl in Connewitz als auch im Leipziger Osten als Reaktion auf 
Räumungen kurzzeitig besetzter Häuser Barrikaden gebaut und angezündet. Es kam zu Auseinandersetzungen mit der Polizei. Eine weitere - auch in der linken Szene umstrittene - Aktion war der gewalttätige Übergriff auf eine Immobilienmaklerin Ende 2019. Doch verprügelte Immobilienmakler:innen und brennende Bagger sind nur ein Teil der wohnungspolitischen Bewegung, auch wenn sie medial die größere Aufmerksamkeit bekommen.

Seit einigen Jahren unterstützen wohnungspolitische Gruppen von Entmietung betroffene Mieter:innen, klären über grundlegende Rechtsansprüche auf und tragen das Thema Wohnen als soziale Frage in die Öffentlichkeit. Eine der ältesten Gruppen ist das Netzwerk „Leipzig - Stadt für Alle“ (SfA), welches sich 2012 im Rahmen der ersten bekannten Entmietungsfälle gründete und seitdem für eine demokratische und soziale Stadtentwicklungs- und Wohnungspolitik kämpft (Leipzig - Stadt für alle 2020). Das Netzwerk unterstützt einerseits betroffene Mieter:innen, stellt andererseits aber in Mitteilungen und auf Veranstaltungen grundlegende Forderungen.

Darüber hinaus gibt es sowohl stadtteilbezogene Initiativen wie Mieter:innengemeinschaften von bedrohten Häusern, zum Beispiel in der Thierbacher Straße 6 (Loch 2018) oder Kantstraße (Nagel 2020), die seit Jahren gegen ihre Verdrängung aus den Häusern und Stadtteilen kämpfen. Auch ist Leipzig bekannt für seine subkulturellen Räume, die sich in verhältnismäßig vielen alternativen Wohnprojek-

Abb. 4 Protest in Coronazeiten in Leipzig. (C) Lina Hurlin)




ten manifestieren, etwa Wagenplätze, Projekträume, Mietshäuser Syndikat-Projekte oder Genossenschaften. Doch auch von diesen schwinden im Zuge fortschreitender Gentrifizierung die, die es nicht geschafft haben, sich rechtlich abzusichern. Die steigenden Miet- und Kaufpreise machen es immer schwieriger, solche Orte neu zu schaffen (Abb. 4).

Die Formen des Widerstands gegen steigende Mieten und Verdrängung sind in Leipzig sehr unterschiedlich in Ausdrucksformen, beteiligten Akteur:innen und auch hinsichtlich der Ausrichtung auf lokale Betroffenheit oder größere politische Forderungen. Auch wenn es teilweise Unstimmigkeiten über Militanz und politische Ausrichtungen gibt, so eint sie doch alle das Ziel der Dekommodifizierung der Wohnraumversorgung und der wachsende Druck auf den Wohnungsmarkt in Leipzig.

\section{Strategische Tendenzen und kontroverse Spannungsfelder in der wohnungspolitischen Bewegung}

Im Austausch über die jüngeren Ereignisse und Veränderungen der wohnungspolitischen Auseinandersetzungen sind uns drei übergeordnete Tendenzen aufgefallen, die sich in allen vier Städten beobachten lassen, sowie zwei prägende Spannungsfelder, die in und zwischen Initiativen in den letzten Jahren immer wieder neu verhandelt werden. Die Entwicklungstendenzen und Konflikte sind nicht durchweg neu für (städtische) soziale Bewegungen (siehe etwa Birke 2014; Mayer 1999). Im Gegenteil lassen sich Institutionalisierungs- und Professionalisierungstendenzen wohl in den meisten Bewegungszyklen beobachten. So erinnert etwa die Debatte in der stadtpolitischen Bewegung der 1980er Jahre über die Vereinnahmung durch staatliche Förderung an heutige Ambivalenzen, die sich aus dieser Entwicklungstendenz ergeben (Mayer 1987). Auch knüpfen heutige Tendenzen des Organizing indirekt an ältere Debatten der Gemeinwesenarbeit in sozialen Bewegungen an. Wenn wir also davon sprechen, dass es sich um „neue“ Entwicklungstendenzen handelt, meinen wir damit „,neu“ für den jetzigen wohnungspolitischen Bewegungszyklus.

\subsection{Tendenz 1: Organizing als neue Strategie der wohnungspolitischen Bewegung}

(Community) Organizing steht für die gezielte Strategie der Ansprache und Einbindung Betroffener in die politische Arbeit (Maruschke 2014). Dabei wird nicht darauf gehofft, dass sich von einem Problem oder gesellschaftlichem Missstand Betroffene spontan äußern oder zusammentun. Vielmehr geht die Strategie des Organizing davon aus, dass Organisierungsprozesse von Personen oder Gruppen angestoßen und mit gezielten Mechanismen am Laufen gehalten werden müssen - und dies mit der Absicht, Macht umzuverteilen: „Community Organizing hat dabei zum Ziel, Macht zu gewinnen, um konkrete, von den BewohnerInnen identifizierte, Interessen zum Wohle des Gemeinwesens umzusetzen“ (Rothschuh 2013, S. 375).

Diese - für die wohnungspolitische Bewegung neu aufgegriffene - Strategie wurde von den US-amerikanischen Bürgerrechts- und gewerkschaftlichen Bewegungen 
Mitte des 20. Jahrhunderts ausformuliert. In der BRD ist die Geschichte wesentlich jünger und taucht hauptsächlich als Strang der Gemeinwesenarbeit in der Sozialen Arbeit auf, wird aber auch von Gewerkschaften angewendet. Dabei lässt sich unterscheiden zwischen Ansätzen, die schnell konkrete Verbesserungen im Alltag erzielen möchten; und jenen, die sich einem transformativen Community Organizing verschreiben und dafür auf Basis einer revolutionären Zukunftsvision eine gesellschaftliche Machtumverteilung durch neue strategische Bündnisse anstreben (Maruschke 2014; Williams 2013; s. dazu unsere Analyse zum Spannungsfeld 2: Zwischen konkreten Zielen und großen Transformationen).

In der wohnungspolitischen Bewegung wurde das (transformative) Community Organizing als bewusste Strategie früh in Hamburg im Protest gegen den Abriss der Esso-Häuser 2013 und in Berlin bei der Initiative „Kotti \& Co“ seit 2011 genutzt (Kotti \& Co 2016; Straßburger 2018). Durch Haustürgespräche, Infostände und eine konstante Präsenz vor Ort wurden in beiden Fällen Mieter:innen wehrhaft, denen vorher eingebläut wurde, das Problem der Verdrängung sei ein individuelles für das sie selbst verantwortlich seien, und deren politische Partizipationsmöglichkeiten vielfältig eingeschränkt waren und z. T. noch sind. In beiden Bündnissen sind vorher nichtorganisierte Mieter:innen prägend, die sich mit Impulsen des Community Organizing selbst ermächtigt haben.

In den letzten Jahren nimmt der explizite Bezug auf den Organizing-Ansatz in der wohnungspolitischen Bewegung zu. Er findet zum Beispiel Anwendung bei der „Mieter:innengemeinschaft Schönefelder Höfe“ in Leipzig gegen Ungerechtigkeiten des Immobilien-Unternehmens Vonovia, um z. B. gemeinsam auf falsche Betriebskostenabrechnungen zu reagieren (Reichle und Bescherer 2021). In Jena verfolgt die „BI Soziales Wohnen“ Ansätze des Organizing in Lobeda durch Haustürgespräche und Besuche in den Häusern. Dies ist zwar nicht systematisch als Community Organizing durchgeplant, funktioniert aber als ein organischer Ansatz, der sich den Verhältnissen vor Ort in Jena einfügt. In Berlin ist die Vernetzung der Deutsche Wohnen-Mieter:innen aus Siedlungen aus ganz Berlin teils selbst organisiert, teils von außen durch organisierende Aktivist:innen angestoßen (Strobel 2020). Diese Initiativen sind nun auch größtenteils in der „Kampagne Deutsche Wohnen und Co. enteignen“ aktiv. Zudem ist aus diesem Kontext die „Starthilfe AG“ entstanden, die mit einer Broschüre (AG Starthilfe 2019) und Schulungen ihr Organizing-Wissen weitergibt - in einem digitalen Stammtisch auch über Berlin hinaus. Auch in Hamburg wird kollektiver Protest durch Organizing immer häufiger. Jüngst organisierten sich die Mieter:innen einer Häuserzeile am Kleinen Schäferkamp erfolgreich, um eine Zwangsversteigerung an die Meistbietenden zu verhindern - und problematisierten dabei über ihr Projekt hinausgehend, dass das Vorkaufsrecht in Milieuschutzgebieten bei Zwangsversteigerungen nicht gilt (Hirschbiegel 2021).

Die planvolle und zeitaufwändige politische Strategie wird seit einigen Jahren auch rege auf den jährlichen Recht-auf-Stadt Foren oder in einzelnen Workshops diskutiert und findet so ihre Verbreitung in der Bewegung. 


\subsection{Tendenz 2: Expertise, Institutionalisierung und Professionalisierung}

In sozialen Bewegungen versammelt sich im Laufe der Zeit immer mehr Expertise. Zunächst wurzeln Bewegungen oftmals in unterschiedlichen individuellen Betroffenheiten (Köhler und Wissen 2003). Um dann jedoch die kritisierten Prozesse wie Spekulation, Zwangsräumungen oder Privatisierungen tatsächlich verhindern zu können, ist Expertise auf mehreren Ebenen nötig. Dazu gehören juristisches Wissen, z. B. um mietrechtliche Instrumente; Kommunikationswissen, z. B. für eine wirksame Öffentlichkeitsarbeit oder um mit Politiker:innen ins Gespräch zu kommen; oder, wie am Beispiel Organizing bereits deutlich geworden ist, methodisches Wissen der Gemeinwesenarbeit, um Kräfte zu bündeln; auch strategisches und fachliches Wissen sind nötig, um den kritisierten Verhältnissen eine positive Alternative entgegenzusetzen.

Die wohnungspolitische Bewegung zeichnet aber nicht nur aus, dass immer mehr fachliche Expertise unter den Aktivist:innen verfügbar ist. Eine sukzessiv zunehmende Relevanz von Fachwissen führt zu einer ambivalenten Institutionalisierung und Professionalisierung sozialer Bewegungen. Als Institutionalisierung bezeichnen wir die regel- bis standardmäßige Einbindung wohnungspolitischer Initiativen in formale Prozesse der Stadtentwicklung. So war z. B. in Leipzig ,SfA“ Teil der Expert:innenrunde für die Entwicklung eines wohnungspolitischen Konzepts der Stadt. Die „Planbude“ aus Hamburg-St. Pauli agierte als Sachverständige für die Jury im Städtebaulichen Wettbewerb um die Neuplanung eines Gebäudeensembles an der Reeperbahn. In Berlin wurden Vertreter:innen der stadtpolitischen Bewegung eingeladen, an der Ausarbeitung der neuen Richtlinien für Bürger:innenbeteiligung in der Stadtentwicklung mitzuarbeiten. Und in Jena wurden zwei Mitglieder der ,JBGInitiative" in den Vorstand der Jenaer Baugenossenschaft gewählt und nehmen so direkt Einfluss auf die Unternehmenspolitik. Eine solche Institutionalisierung zeugt von der errungenen politischen Macht und von der Fachlichkeit der wohnungspolitischen Bewegung.

Je länger die wohnungspolitische Bewegung existiert, desto größer wird der Drang nach Verstetigung und damit auch Professionalisierung. Das zumindest lassen neuere Entwicklungen in einigen der von uns diskutierten Städte erkennen. Hier lassen sich zwei Formen der Professionalisierung erkennen. Erstens wurden Stellen durch die lokalen Regierungen eingerichtet und finanziert, die Partizipation organisieren, alternative Wohnprojekte beraten, die wohnungspolitische Bewegung vernetzen oder Wissen weitertragen sollen. Beispiele dafür sind das „Initiativenforum“ auf Landes- und die Arbeits- und Koordinierungsstruktur „Gemeinwohlorientierte Stadtentwicklung" (AKS-Gemeinwohl) auf Bezirksebene in Berlin bzw. FriedrichshainKreuzberg oder das „Netzwerk Leipziger Freiheit“, das als Beratungs- und Vernetzungsstelle für Wohnprojekte kooperative Wohnformen fördert. In Hamburg gibt es zwar bereits seit Ende der 1990er Jahre die „Hamburger Wohn-Projektetage“ von STATTBAU; die Stadt ergänzt das Beratungs- und Vernetzungsangebot aber seit einiger Zeit selbst durch eine „Agentur für Baugemeinschaften“. Darin ist eine Tendenz der Verwässerung und Vereinnahmung abzulesen (denn Baugemeinschaften tragen oftmals nicht zu einer sozialen Wohnraumversorgung bei), die in Jena noch übertroffen wurde: Hier wurde entgegen der Kritik aus der Bewegung ein „Ci- 
ty Manager" engagiert, dessen vorrangige Aufgabe es ist, eine Governance für die Attraktivitätssteigerung der Innenstadt zu sichern (Imagekampagnen, Leerstandsaktivierung).

Zweitens wird die Bewegung zunehmend von professionalisierten Strukturen getragen. So verfassen sich viele Initiativen formal als Verein oder Genossenschaft und geben sich Statuten, in denen z.B. Entscheidungsabläufe verbindlich festgelegt werden. Nach vielen Jahren im Kampf um alternative Wohnungspolitiken sind es formalisierte Einrichtungen wie Gemeinwesenarbeit, Stadtteilbüros und Mieter:innenvereine, in denen Personen einen Teil ihrer Zeit entlohnt für die Organisation der Bewegung aufwenden, sie am Leben halten oder neu beflügeln können. Die Mieten-Volksinitiativen in Hamburg gehen z. B. auf Initiative der Mietervereine und wenigen Mitstreiter:innen mit viel politischem und juristischem Fachwissen zurück. In Berlin verfügen z. B. die Initiative „Stadt von Unten“ mit der „ZusammenStelle“ und „Kotti \& Co“ mit der Durchführung von Studien durch Förderungen von Bezirk bzw. Senat über Mittel, mit denen einzelne Mitglieder der Gruppen ihre politische Arbeit professionalisiert haben. In Jena liefert der Verein „Faires Wohnen in Jena" alternative Wohnungsmarkt-Analysen zum kommunalen Monitoring und somit argumentatives Futter für die Bewegung; der Verein arbeitet hierfür aber gemeinnützig und ohne externe Förderung. In Thüringen beraten und vernetzen zudem die „Wohnstrategen“ alternative Baugruppen und Wohnprojekte.

Die verstärkte Institutionalisierung und Professionalisierung sind jedoch ambivalent. Einerseits tragen diese Entwicklungen zur Kontinuität der Bewegung bei; denn sie sichern sowohl Finanzierung als auch Qualifizierung (s. bereits Mayer 1987). Die zunehmende Einbindung in formale Politiken, die mit der Einrichtung bezahlter Stellen einhergehen kann (aber nicht muss), kann andererseits aber auch zu einer Verwässerung oder sogar Vereinnahmung der Forderungen führen (Mayer 2009). Durch Formalisierung und Professionalisierung entstehen außerdem Wissenshierarchien und Netzwerkhierarchien, die zu Machtungleichgewichten innerhalb der Bewegung führen. Gleichzeitig bieten sie den Vorteil, dass Bewegungswissen nicht von jeder Gruppe neu erarbeitet werden muss, sondern weitergegeben werden kann. Die mit bezahlten Stellen einhergehenden Zeitressourcen sind außerdem oft Voraussetzung, um sich überhaupt auf ressourcenintensive Prozesse wie kooperative Planungen einlassen zu können. Von staatlicher Seite finanzierte Stellen sind aber der Gefahr ausgesetzt, radikalere Teile der Bewegung auszuschließen und die Bewegung insgesamt in staatliche Wohnungspolitiken einzupassen. Weitreichende Forderungen nach bezahlbarem Wohnraum für alle, mehr Mitbestimmung und völlig neue Eigentumsstrukturen können in dem Zuge auch in Kompromissen bis zu Unkenntlichkeit verändert werden. Diese Vor- und Nachteile der Institutionalisierung und Professionalisierung werden in der wohnungspolitischen Bewegung bisher nur in Ansätzen reflektiert.

\subsection{Tendenz 3: Vernetzung und Kooperation}

Die wohnungspolitische Bewegung der vier Städte ist durch ihre lokale Ausrichtung geprägt: Initiativen entstehen meist auf Haus- oder Siedlungsebene und haben dementsprechend (zunächst) einen kleinräumigen Bezug, sowohl in ihrer thematischen 
Ausrichtung als auch in ihrer Organisationsform. Um als soziale Bewegung Wirkung - in Form von medialer Aufmerksamkeit oder Einfluss auf institutionelle Politik zu erlangen, ist es aber zentral, dass diese lokalen Gruppen sich vernetzen und mit anderen Akteuren kooperieren. Solche Vernetzungen und Kooperationen verstärken sich in jüngster Zeit (Rink und Vollmer 2019).

Die Vernetzung in Form von Austausch und Zusammenarbeit einzelner wohnungs- und stadtpolitischer Initiativen vollzieht sich auf verschiedenen Ebenen: auf der nachbarschaftlichen, in stadtweiten Netzwerken, im deutschland- oder europaweiten Austausch. Auf nachbarschaftlicher Ebene sind z. B. im Berliner Wrangeloder Reichenbergerkiez enge Netzwerke zwischen verschiedenen Initiativen entstanden. In Hamburg gibt es die Plattform „St. Pauli Selbermachen“, die eine Stadtteilzeitung herausgibt und Stadtteilversammlungen organisiert. In Leipzig ist als lokale Initiative für den Leipziger Süden die „Vernetzung Süd“ entstanden, die sich zum Beispiel auch mit den Entmietungen in der Kantstraße und Thierbacher Straße beschäftigt. In Jena planen die BI und die JBG-Initiative gemeinsam eine aktivierende Mieter:innenumfrage in den jeweiligen Beständen. Stadtweite Netzwerke existieren in allen vier Städten: In Jena und Hamburg unter dem Namen Recht auf Stadt, in Berlin mit dem Mietenwahnsinn-Bündnis und in Leipzig mit SfA. Es variiert jedoch zwischen den Städten und zu unterschiedlichen Zeiten, wie hoch der Anteil der darin vernetzen Initiativen an der gesamten Bewegungslandschaft ist und wie hoch der Aktivitätsgrad der Netzwerke ist.

Die bundesweite Vernetzung hat sich in den letzten Jahren merklich intensiviert. Das jährliche Recht auf Stadt-Forum fand bereits acht Mal statt, jeweils in einer anderen Stadt, darunter Hamburg, Leipzig und Weimar unter Beteiligung von Gruppen aus Jena. Auf ihm tauschen wohnungs- und stadtpolitische Initiativen ihre Erfahrungen aus und schmieden zunehmend Pläne für bundesweite Kampagnen. Daraus entstehen auch Impulse für Solidaritätsbekundungen oder sogar konkret Unterstützung von Initiativen in anderen Städten (Abb. 1). Auch die prinzipielle Debatte um Bedingungen für alternative Miet- und Wohnformen ist deutschlandweit zunehmend im „Netzwerk Mieten \& Wohnen“ koordiniert, das z. B. Konferenzen organisiert oder einen Blog unterhält. Eine weitere wichtige koordinierte Protestform sind dezentrale, aber gleichzeitige Demonstrationen oder Aktionen, wie am jährlichen „Housing Action Day“, an dem sich zahlreiche Städte in Deutschland beteiligen und der auch europaweit abgestimmt mit der „European Action Coalition for the Right to Housing and to the City" stattfindet.

Neben der in den letzten Jahren intensivierten Zusammenarbeit und Vernetzung von wohnungspolitischen Initiativen untereinander lässt sich auch eine verstärkte Kooperation mit anderen, Nichtbewegungsakteuren der Zivilgesellschaft, die sich im Themenfeld Wohnraumversorgung betätigen, feststellen. In Berlin und Hamburg arbeiten z. B. die Mieter:innenvereine bzw. „Mieter helfen Mieter“ mit basisgetragenen Mieter:inneninitiativen zusammen. In Leipzig wird der „Mieterverein“ immer mehr auch von Aktiven aus der Bewegung genutzt, um wohnungspolitische Interessen einzubringen. In Jena dagegen kam eine Zusammenarbeit mit dem Mieterverein trotz einiger Kooperationsangebote aus der Bewegung (bspw. Mietparade 2019 und 2020, s. Abb. 3) bisher nicht zu Stande. 
Auf Bundesebene liegt beim Deutschen Mieterbund (DMB) ein Beschluss vor, der vorsieht, die Zusammenarbeit mit der Bewegung zu intensivieren. Dies hat sich zum Beispiel in einem gemeinsam von DMB und wohnungspolitischen Initiativen organisierten „Mietengipfel“ im Jahr 2018 als Gegenveranstaltung zum von Bundesbauminister Seehofer veranstalteten Gipfel geäußert. Daran waren auch Gewerkschaften und Sozialverbände beteiligt, die in den letzten Jahren verstärkt zu dem Thema aktiv geworden sind. Diese Akteurskonstellation hat sich mit dem „Bündnis Wohnen ist Menschenrecht“ im Jahr 2019 institutionalisiert. Die Zusammenarbeit mit diesen anderen zivilgesellschaftlichen Akteuren bringt auch Konflikte mit sich, da die Handlungsrationalitäten einer sozialen Bewegung und der stärker institutionalisierten, langfristig ausgerichteten Vereine und Verbände sich oftmals beißen (Vollmer 2021).

\subsection{Spannungsfeld 1: Zwischen Einhegung und Selbstermächtigung}

Während sich die Bewegung für eine substanzielle Demokratisierung der Stadtpolitik und Möglichkeiten, sich selbst zu organisieren, einsetzt, gibt es zugleich immer mehr offizielle Partizipationsangebote. Diese wirken allerdings oft eher einhegend oder gar vereinnahmend. Dieses Spannungsfeld wird in der Bewegung kontrovers diskutiert, und den Gefahren wird mit unterschiedlichen Mitteln begegnet.

Das Baugesetzbuch sieht vor, dass öffentliche Planung mit Instrumenten der formellen wie informellen Beteiligung stattfindet. Über Stadtteilbüros, Quartiersmanagement und andere Anlaufstellen in den Stadtteilen, zumeist finanziert durch die Städtebauförderung, können Bewohner:innen ihre Anliegen artikulieren. Die Möglichkeit zur Mitsprache wird somit gewährleistet; für Protest und Gegenstimmen zum herrschenden Diskurs ist allerdings oft kein Platz. Bei vielen dieser Einrichtungen wird kompromissorientiert agiert, d.h. Konflikte werden unparteiisch verwaltet und so entschärft. Die systematischen Ursachen von Problemen werden nur selten thematisiert (Lanz 2000). In einzelnen Fällen sowie je nach Finanzierung und Politisierung der Mitarbeiter:innen dieser Einrichtungen können in Quartiersbüros auch kritische Stimmen zur Stadtentwicklung gehört werden. Am ehesten geschieht dies noch in den von den Büros organisierten Stadtteilbeiräten, wie z. B. der ,Stadtteilbeirat St. Georg“ in Hamburg oder dem „Quartiersrat Schöneberger Norden“ in Berlin.

Die Mitbestimmung über letztlich entscheidende Fragen (Bezahlbarkeit, Mietpreise, Verdrängungsmechanismen) findet in diesen Gremien nicht statt. Anwohner:innen können über diese Institutionen mit Stellungnahmen und Gegenexpertise intervenieren, zumindest wenn sie über die notwendigen Zeitressource verfügen, erhöhen dadurch aber kaum den Handlungsdruck. Somit besteht die Gefahr der Vereinnahmung der Bewegung durch solche Partizipationsangebote.

Fehlende Mitwirkungsmöglichkeiten oder intransparente formelle Beteiligungsverfahren sind oft Anlass von Protest. Die wohnungspolitische Bewegung kann Beteiligungsverfahren boykottieren, kapern, ihre Demokratisierung in kooperativer Form fordern oder eigene Planungen gegen die offiziellen Stellen voranbringen. Der Konflikt um den Eichplatz in Jena ist ein Beispiel für die erzwungene Demokratisierung eines vorher top-down geplanten Beteiligungsverfahrens. In einer breitan- 
gelegten Kampagne aus parlamentarischen Stimmen aus dem Stadtrat und außerparlamentarischen Stimmen wurden kooperative Bürger:innenbeteiligungsformate wie Werkstätten und Runde Tische durchgesetzt. In Berlin boykottierten Initiativen zum Beispiel die Partizipationsveranstaltungen zur Bebauung des Tempelhofer Feldes, nachdem ersichtlich wurde, dass die generelle Bebauungsfrage nicht teil der Mitbestimmung sein sollte - teils kaperten sie die offiziellen Veranstaltungen mit eigenen Formaten (Hilbrandt 2016). In Leipzig gelang es nach Protest einer Initiative zum Erhalt eines dort befindlichen Wagenplatzes und für die Einrichtung eines selbstorganisierten Stadtteilpark die Überarbeitung des Bebauungsplans einer Brachfläche im Leipziger Osten in einem Beteiligungsverfahren anzustoßen (Julke 2020). In Hamburg ist es gelungen, eine Olympiabewerbung abzuwehren und damit langfristig zumindest die Grundlage dafür zu schaffen, statt des olympischen Geländes auf dem Grasbrook einen neuen Stadtteil mit sozialer Wohnraumversorgung zu entwickeln.

Diese Fälle zeigen: Gerade, wenn die Privatisierung von Grundstücken verhindert oder rückgängig gemacht werden konnte, gelang es den Bewegungen, lokalstaatliche Akteure dazu bewegen, ihnen weitgehende Mitbestimmungsrechte in der Entwicklung dieser Grundstücke einzuräumen. Diese kooperativen Verfahren durchzusetzen, kann auch dann eher gelingen, wenn die Initiativen selbst konkrete Ideen und Pläne als eine Art Gegenplanung vorlegen.

Auch direktdemokratische Verfahren wie Volksentscheide, Einwohner:innenanträge und Bürger:innenbegehren werden in allen vier Städten durch die wohnungspolitische Bewegung als Erweiterung der offiziellen Bürger:innenbeteiligung angewandt. Diese Protestformen ermöglichen es, Mieter:innen mit einem konkreten Anliegen ansprechen zu können, öffentliche Debatten zu bestimmten Themen anzustoßen und - bei Erfolg - konkrete materielle Verbesserungen auch durchzusetzen. Mit all diesen Strategien - der Ausweitung von Beteiligungsmöglichkeiten durch Protest, durch ,selber machen“ oder durch direktdemokratische Instrumente - ermächtigen sich die wohnungspolitischen Bewegungsakteure selbst.

\subsection{Spannungsfeld 2: Zwischen konkreten Zielen und großen Transformationen}

In der wohnungspolitischen Bewegung wird kontrovers debattiert, welcher der kürzeste oder realistischste Weg zum Fernziel der Dekommodifizierung und Vergesellschaftung der Wohnraumversorgung ist. In den jährlich stattfindenden „Recht-aufStadt-Foren" kristallisieren sich zwei strategische Richtungen heraus: zum einen der munizipalistische Weg, d.h. der Versuch, mittels lokaler progressiver Bündnisse aus Bewegung und Parteien ein kommunales Gegengewicht in den Parlamenten zur zentralstaatlichen Regulation aufzubauen und von außen Druck auf diese lokalen Parlamente auszuüben; zum anderen der syndikalistische Weg, d.h. mittels autonomer Initiativen aus betroffenen Mieter:innen deren Interessen direkt gegenüber den Wohnungsunternehmen oder Vermieter:innen durchzusetzen.

Diese theoretisch geführte strategische Debatte bricht sich in der Praxis jedoch allenfalls indirekt Bahn. Hier stehen in der Regel Organisierungsfragen im Vordergrund, die in der Praxis zu strategischen Mischformen führen. Zwischen und 
selbst innerhalb von einzelnen Initiativen ist nicht strikt danach zu unterscheiden, wer überwiegend Selbsthilfe praktiziert oder überwiegend übergeordnete Forderungen artikuliert. Idealtypisch lassen sich dennoch unterschiedliche strategische und organisatorische Ansätze identifizieren.

Was Hannah Müller und Lisa Vollmer (2020) für die Thüringer Mieter:innenbewegung beobachten, lässt sich auf die vier hier untersuchten Städten übertragen: Auf der einen Seite versuchen Initiativen, ausgehend von einer abstrakten Kritik an der herrschenden Wohnraumversorgung, stadtweite Diskurse zu beeinflussen. Auf Basis eines zumeist antizipierten ideellen Gesamtinteresses der Mieter:innen werden Groß-Demos organisiert, Bündnisse zwischen etablierten Akteuren geschmiedet und mit advokatischer Expertise die Ausrichtung städtischer Wohnungspolitiken mit Pressemitteilungen oder Manifesten kritisiert. Beispiele aus unseren Städten sind das 10-Punkte-Papier von RaS Hamburg für eine andere Wohnraumversorgung für Geflüchtete oder der Forderungskatalog von Leipzig SfA für die verstärkte Nutzung des Vorkaufsrechts in Leipzig. Der Aktionsradius beläuft sich in der Regel auf die gesamte Stadt. Die eigene Betroffenheit von Wohnungsproblemen spielt für das Engagement eine untergeordnete Rolle.

Auf der anderen Seite stehen Initiativen, die von konkreten Verletzungen von Mieter:inneninteressen ausgehend versuchen, diesen zur Durchsetzung zu verhelfen. Die eigene Betroffenheit ist Ausgangs- und Kontinuitätsfaktor für das Engagement, und auf dieser Basis werden weitere Mieter:innen mittels Organizing mobilisiert. Die Initiativen sind sehr heterogen und setzen sich aus Aktiven verschiedener Schichten bzw. Communities zusammen. Daraus entstehen zum einen Initiativen, die sich auf Basis eines Problems vermieterübergreifend zusammenfinden, wie bspw. „Miettreff“ aus Jena, der „Miettreff Leipziger Osten“, „Zwangsräumung verhindern“ aus Berlin oder das „Aktionsbündnis gegen Wohnungsnot“ in Hamburg, das Wohnungslosigkeit problematisiert. Zum anderen organisieren sich Initiativen entlang eines Vermieters, wie z.B. die Vernetzung der „Akelius-Mieter:innen“ in Berlin und Hamburg, die BI gegenüber jenawohnen oder die „Mietergemeinschaft Schönefelder Höfe“ in Leipzig gegenüber Vonovia.

Weiterhin kann zwischen Initiativen unterschieden werden, die bewusst nur auf direkte Aktionen setzen, um das Problem im Sinne der Mieter:innen ohne parlamentarische „Umwege“ direkt durch Aufbau von Druck gegenüber den Vermieter:innen zu lösen; und Initiativen, die Mieter:innen organisieren, um damit kommunalpolitische Mehrheiten für mieter:innenfreundliche wohnungspolitische Instrumente zu organisieren, wie in Teilen die BI. Zum ersten Typus zählen Initiativen wie „Wilhelmsburg Solidarisch“ oder das Rom:nija-Beratungscafé „Romani Kafava“ aus Hamburg (die sich allerdings beide nicht nur zur Wohnungsfrage solidarisieren, sondern auch zu Arbeit, Behördenkontakt etc.) oder die „Mieter:innengewerkschaft“ in Berlin; zum ersten Typus zählen aber auch kleinere Hausgemeinschaften, die entweder bereits über selbst genutztes und demokratisch verwaltetes Eigentum befinden (wie Mietshäuser-Syndikatsprojekte) oder dieses anstreben.

Solche idealtypischen Unterscheidungen dienen vor allem einer Analyse von Praxen und den dahinter liegenden strategischen und organisatorischen Positionen. In der Realität existieren sie nicht als Reinform. So haben viele Initiativen, denen es zunächst darum ging, den allgemeinen Diskurs zu beeinflussen, Arbeit mit konkret 
Betroffenen in ihre politische Praxis integriert. Umgekehrt gelingt es vielen Initiativen, die aus der unmittelbaren Betroffenheit heraus agieren, davon zu abstrahieren und allgemeine diskursive Verschiebungen zu erwirken. Auch die Unterscheidung zwischen der Strategie, Druck entweder auf Vermieter:innen oder die Politik auszuüben, ist in der Praxis selten in Reinform anzutreffen. Druck auf Vermieter:innen wird z.B. auch dadurch aufgebaut, dass lokalstaatliche Steuerungsinstrumente genutzt werden. Die dargestellte strategische Polarisierung ist also in der Praxis der wohnungspolitischen Bewegung deutlich weniger stark ausgeprägt, als so manche Diskussion darüber den Anschein erweckt.

\section{Aktuelle Herausforderungen für die wohnungspolitische Bewegung - ein Ausblick}

Das Thema Wohnraumversorgung hat - auch dank der Aktivität der wohnungspolitischen Bewegung - an Dringlichkeit und gesellschaftlicher Aufmerksamkeit gewonnen. Auch wenn die institutionelle Politik sich stärker als noch vor einigen Jahren zu Mieter:inneninteressen hinwendet, bleibt die zentrale Forderung der Bewegung weitgehend unerfüllt: Immer noch wird Wohnraum als Ware gehandelt, eine Dekommodifizierung und Vergesellschaftung der Wohnraumversorgung scheint noch in weiter Ferne. Die COVID-19-Pandemie hat die negativen Auswirkungen der warenförmigen Organisation der Wohnraumversorgung und die Verbindung der Wohnungsfrage mit sozialer Ungleichheit wieder einmal wie unter einem Brennglas gezeigt. Die Mietbelastung ist für viele wegen Einkommenseinbußen gestiegen. Die ungleiche Verteilung von qualitätsvollem Wohnraum hat darüber bestimmt, wer in Zeiten von Ausgangssperren, Homeoffice und Homeschooling seinen Alltag räumlich erträglich organisieren konnte. Besonders hart waren diejenigen betroffen, die keine Wohnung haben oder in ihrer Wohnung häuslicher Gewalt ausgesetzt sind.

So, wie die Pandemie die Probleme der kapitalistischen Wohnraumproduktion offengelegt hat, so verweist sie auch auf einige Herausforderungen der wohnungspolitischen Bewegung, die es aus unserer Sicht zu meistern gilt. Die in der Pandemie zu beobachtende Hinwendung zur Nachbarschaft als sozialem Raum mit den zahlreichen Nachbarschaftsinitiativen, die Unterstützung bei Einkäufen, anderen Besorgungen oder Kinderbetreuung für Risikogruppen und Menschen in Quarantäne anbieten, verweist darauf, dass die Wohnungsfrage nicht isoliert betrachtet werden darf. Sie ist eng mit Fragen der Gesundheitsversorgung, mit ökologischen Fragen, Fragen rassistischer Exklusion und feministischen Fragestellungen verbunden. All diese Fragen stellen und materialisieren sich ganz konkret in der Alltagsbewältigung im lokalen Raum der Nachbarschaft, werden aber bisher von der wohnungspolitischen Bewegung nur punktuell mit den eigenen Forderungen verbunden.

Besonders ökonomisch und kulturell marginalisierte Gruppen sind von der Pandemie getroffen. Wie in vielen sozialen Bewegungen, so ist und bleibt es auch in der wohnungspolitischen Bewegung eine Herausforderung, die Interessen dieser Gruppen einzubeziehen sowie Betroffene selbst einzubinden. Obdachlose, Geflüchtete, von häuslicher Gewalt betroffene Frauen sind zwar auf günstigen Wohnraum angewiesen, jedoch kaum in der Bewegung organisiert. 
Auch hat die Pandemie die Rolle der föderalen Struktur der Bundesrepublik verdeutlicht. Diese spielt auch bei der Wohnungsfrage eine zentrale Rolle. Das Kippen des Berliner Mietendeckels durch das Bundesverfassungsgericht hat die sich ohnehin verstärkende Hinwendung der Bewegung zur Bundesebene, auf der zentrale Steuerungskompetenzen der Wohnungspolitik liegen, befeuert. Forderungen nach der Einführung eines bundesweiten Mietendeckels werden im Zuge der bundesweiten Mietenstopp-Kampagne laut, eine erste zentrale bundesweite Demonstration hat im September 2021 stattgefunden.

Und nicht zuletzt kündigt sich zwischen der sozialen Frage nach bezahlbarem und qualitativem Wohnraum für breite Schichten und der ökologischen Frage ein Widerspruch an. Zumindest dessen bisherige staatliche Regulation lässt erahnen, dass die Mieter:innen mehr als die Eigentümer:innen für die ökologische Transformation im Immobiliensektor zur Kasse gebeten werden. Schon leichte Verschiebungen wie die jüngst geplante paritätische Kostenteilung der $\mathrm{CO}_{2}$-Bepreisung sorgen bereits für einen Sturm der Entrüstung bei Lobbyverbänden von Vermieter:innen. Zwar gibt es noch keine überzeugenden Konzepte für eine sozial-ökologische Transformation, die auch die Wohnungsnot im Blick hat - das „Netzwerk Mieten \& Wohnen“ diskutiert aber bereits mögliche Regeln für ein klimagerechtes und bezahlbares Wohnen.

Vielleicht eröffnet die Pandemie - mit ihren drastischen wirtschaftlichen Folgen und dem möglichen staatlichen Eingreifen in Reaktion darauf - auch eine Verschiebung in der Diskussion der Wohnungsfrage: eine Hinwendung zum Wohnen als zentralem menschlichem Bedürfnis und weg von kapitalistischen Interessen in der Wohnraumverwertung. Noch steigen die Mieten und Menschen verlieren ihr Zuhause. Aber es ist in Bewegung auf den Straßen, vor den Laptops und in den Parlamenten. Wir werden weiter beobachten, was das in Berlin, Leipzig, Hamburg und Jena so macht - mit den Häusern und ihren Bewohner:innen.

\section{Literatur}

AG Starthilfe des Mieter*innenprotests Deutsche Wohnen und der Kampagne Deutsche Wohnen und Co enteignen (2019). Zusammen tun! deutsche-wohnen-protest.de/wp-content/uploads/2019/02/ mieterinnen_protest_deutsche_wohnen_broschuere_zusammentun_2019.pdf. Zugegriffen: 22. März 2021.

Amt für Statistik und Wahlen Leipzig (2016). Bevölkerungsvorausschätzung. https://statistik.leipzig.de/ statpubl/index.aspx?cat=13\&rub=1. Zugegriffen: 22. März 2021.

Amt für Statistik und Wahlen Leipzig (2019). Bevölkerungsvorausschätzung. https://statistik.leipzig.de/ statpubl/index.aspx?cat=13\&rub=1. Zugegriffen: 22. März 2021.

Amt für Statistik und Wahlen Leipzig (2021). Bevölkerungsbestand. https://statistik.leipzig.de/. Zugegriffen: 2. Juni 2021.

Birke, P. (2014). Sozialproteste im ,unternehmerischen“ Hamburg. Notizen zu ihrer Geschichte. In N. Gestring, R. Ruhne \& J. Wehrheim (Hrsg.), Stadt und soziale Bewegungen (S. 83-97). Wiesbaden: Springer VS.

Bürgerschaft der FHH (Freie und Hansestadt Hamburg) (2020). Große Anfrage der Abgeordneten Anke Frieling et al. (CDU) und Fraktion vom 30.09.20 und Antwort des Senats, Betreff: Der Hamburger Wohnungsmarkt: Bestand und aktuelle Entwicklung. Drucksache 22/1595

empirica regio (2021). https://go.empirica-regio.de/go-live/. Zugegriffen: 13. Juni 2021.

$\mathrm{F}+\mathrm{B}-$ Forschung und Beratung für Wohnen, Immobilien und Umwelt GmbH (2021). Mietspiegelindex 2020. Hamburg: Eigenverlag.

Göbel, J. (2020). Wissen aneignen, mehr werden, Druck aufbauen Mieter*innenorganisierung in Jena. Comun, 2(3), 38-40. 
Hilbrandt, H. (2016). Insurgent participation. Consensus and contestation in planning the redevelopment of Berlin-Tempelhof airport. Urban Geography, 38(4), 537-556.

Hirschbiegel, T. (2021). Angst vor Spekulanten: Diese Mieter in Hamburg wollen kämpfen. https://www. mopo.de/hamburg/zwangsversteigerung-angst-vor-spekulanten--diese-mieter-in-hamburg-wollenkaempfen-38221048. Zugegriffen: 4. Juni 2021.

Holm, A. (2014). Wiederkehr der Wohnungsfrage. Aus Politik und Zeitgeschichte, 64(20-21), 25-30.

Holm, A. (2020). Berlin: Mehr Licht als Schatten. Wohnungspolitik unter Rot-Rot-Grün. In D. Rink \& B. Egner (Hrsg.), Lokale Wohnungspolitik. Beispiele aus deutschen Städten (S. 43-64). Baden-Baden: Nomos.

IBB - Investitionsbank Berlin (2020). Wohnungsmarktbericht 2019. Berlin: IBB.

Jacobs, T. (2020). Zwischen Markt und Steuerung - Wohnungspolitik in Jena. In D. Rink \& B. Egner (Hrsg.), Lokale Wohnungspolitik Beispiele aus deutschen Städten (S. 291-308). Baden-Baden: Nomos.

Julke, R. (2020). Grüne beantragen für den Stadtteilpark Volkmarsdorf ein neues Planverfahren. https:// www.l-iz.de/politik/brennpunkt/2020/05/Gruene-beantragen-fuer-den-Stadtteilpark-Volkmarsdorfein-neues-Planverfahren-mit-richtiger-Buergerbeteiligung-329565. Zugegriffen: 13. Juni 2021.

Kadi, J., Vollmer, L., \& Stein, S. (2021). Post-neoliberal housing policy? Disentangling recent reforms in New York, Berlin and Vienna. European Urban and Regional Studies. https://doi.org/10.1177/ 09697764211003626.

Kemper, J., \& Vogelpohl, A. (2020). Die sozial-unternehmerische Stadt: Gesellschaftliche Ungleichheit, Reproduktionskrise und Stadtpolitik in Hamburg. Geographica Helvetica, 75, 221-233.

Köhler, B., \& Wissen, M. (2003). Glocalizing protest: urban conflicts and global social movements. International Journal for Urban and Regional Research, 27(4), 942-951.

Kotti \& Co (2016). Alles muss man selber machen. Multi-Layer-Organizing für eine soziale Wohnraumversorgung in Berlin. sub urban, 4(2/3), 121-126.

Lanz, S. (2000). Der Staat verordnet die Zivilgesellschaft. Widersprüche, 20(78), 39-51.

Leipzig - Stadt für alle (2020). Über uns. http://leipzig-stadtfueralle.de/ueber-uns/. Zugegriffen: 13. Juni 2021.

Loch, R. (2018). Protest in der Thierbacher Straße gegen Entmietung. https://www.l-iz.de/leben/gesellschaft/ 2018/07/Protest-in-der-Thierbacher-Strasse-gegen-Entmietung-224737. Zugegriffen: 13. Juni 2021.

Maruschke, R. (2014). Community Organizing. Zwischen Revolution und Herrschaftssicherung. Eine kritische Einführung. Münster: edition assemblage.

Mayer, M. (1987). „Staatsknete“ und neue soziale Bewegungen. In T. Kreuder \& H. Loewy (Hrsg.), Konservativismus in der Strukturkrise (S. 484-502). Frankfurt am Main: Suhrkamp.

Mayer, M. (1999). Städtische soziale Bewegungen. In A. Klein, H.-J. Legrand \& T. Leif (Hrsg.), Neue soziale Bewegungen - Impulse, Bilanzen und Perspektiven (S. 257-270). Wiesbaden, Opladen: Westdt. Verlag.

Mayer, M. (2009). The 'Right to the City' in the Context of Shifting Mottos of Urban Social Movements. City, 13(2/3), 362-374.

Metzger, J. (2020). Auseinandersetzungen um die „Bündnispolitik für das Wohnen“ in Hamburg. In D. Rink \& B. Egner (Hrsg.), Lokale Wohnungspolitik. Beispiele aus deutschen Städten (S. 65-79). Baden-Baden: Nomos.

Müller, H., \& Vollmer, L. (2020). Die Thüringer Mieter*innenbewegung. Bewegungspolitische Praktiken jenseits der Metropolen. Forschungsjournal Soziale Bewegungen, 33(1), 357-368.

Nagel, J. (2020). Mieterinnen und Mieter der Kantstraße 55-63b in der Leipziger Südvorstadt unter massivem Druck. https://www.l-iz.de/melder/wortmelder/2020/11/Mieterinnen-und-Mieter-derKantstrasse-55-63b-in-der-Leipziger-Suedvorstadt-unter-massivem-Druck-360042. Zugegriffen: 13. Juni 2021.

Pott, M. (2020). Wie setzt sich die Hamburger Neubau-Strategie mit Drittelmix durch? Eine Hegemonieanalyse auf der Suche nach Ansatzpunkten für eine soziale Wohnungspolitik. Unveröffentlichte Masterarbeit an der Universität Hamburg

Reichle, L. R., \& Bescherer, P. (2021). Organizing with tenants and fighting rightist resentments. A case study from east Germany. Radical Housing Journal, 3(1), 11-31.

Rink, D., \& Vollmer, L. (2019). „Mietenwahnsinn stoppen!“ Netzwerke und Mobilisierungen der Mieter*innenbewegung in deutschen Großstädten. Forschungsjournal Soziale Bewegungen, 32(3), 337-349.

Rinn, M. (2016). Konflikte um die Stadt für alle - Das Machtfeld der Stadtentwicklungspolitik in Hamburg. Münster: Westfälisches Dampfboot. 
Rothschuh, M. (2013). Community Organizing - Macht gewinnen statt beteiligt werden. In S. Stövesand, C. Stoik \& U. Troxler (Hrsg.), Handbuch Gemeinwesenarbeit (S. 375-383). Opladen: Barbara Budrich.

Sarnow, M. (2019). Wir kaufen den Kiez zurück. Milieuschutz und Vorkaufsrecht als Ansätze einer postneoliberalen Wohnraumversorgung in Berlin Friedrichshain-Kreuzberg? sub urban, 7(1/2), 115-136.

Schönig, B., Schipper, S., \& Kadi, J. (Hrsg.). (2017). Wohnraum für alle?! Perspektiven auf Planung, Politik und Architektur (S. 105-130). Bielefeld: transcript.

Stadt Jena (2020). Wohnstadt Jena. Stadtumbau und kommunale Wohnraumversorgung. Jena: Eigenverlag.

Statistische Ämter des Bundes und der Länder (2021). Einkommensverteilung (Kreise). https://www. statistikportal.de/de/vgrdl/ergebnisse-kreisebene/einkommen-kreise. Zugegriffen: 13. Juni 2021.

Straßburger, J. (2018). Konfliktlösung in der Stadtentwicklung durch partizipative Planung und GWA am Beispiel des Esso-Häuser-Konflikts. Bachelorarbeit, Hochschule für Angewandte Wissenschaften Hamburg. https://reposit.haw-hamburg.de/bitstream/20.500.12738/8645/1/2019Strassburger_Jan. pdf. Zugegriffen: 17. Nov. 2020.

Strobel, H. (2020). Organisiert gegen einen profitorientierten Wohnungskonzern. sub\urban, 8(3), 195-204.

Tappert, A. (2019). Wohnen muss bezahlbar bleiben - 3000 Leipziger gehen auf die Straße. https://www. 1vz.de/Leipzig/Lokales/Wohnen-muss-bezahlbar-bleiben. Zugegriffen: 13. Juni 2021.

TMIL - Thüringer Ministerium für Infrastruktur und Landwirtschaft (Hrsg.). (2019). Wohnungsmarktbericht Thüringen. Erfurt: Eigenverlag.

Vittu, E. (2021). Recht auf Stadt. Von einem theoretischen Konzept aus Frankreich zu „Recht auf Stadt“Bewegungen in Lateinamerika und Europa. Weimar: IfEU Edition.

Vogelpohl, A., \& Buchholz, T. (2017). Breaking with neoliberalization through restricting the housing market: novel urban policies and the case of Hamburg, Germany. International Journal for Urban and Regional Research, 41(2), 266-281.

Vogelpohl, A., Vollmer, L., Vittu, E., \& Brecht, N. (2017). Die Repolitisierung des Wohnens. Städtische soziale Bewegungen für ein Recht auf Wohnen und auf Stadt in Hamburg, Berlin, Jena und Leipzig. In B. Schönig, S. Schipper \& J. Kadi (Hrsg.), Wohnraum für alle?! Perspektiven auf Planung, Politik und Architektur (S. 105-130). Bielefeld: transcript.

Vollmer, L. (2018). Strategien gegen Gentrifizierung. Stuttgart: Schmetterling.

Vollmer, L. (2019a). Der Gentrifizierungsbegriff in wohnungspolitischen Protesten. sub urban, 7(3), 113-118.

Vollmer, L. (2019b). Mieter_innenbewegungen in Berlin und New York. Die Formierung politischer Kollektivität. Wiesbaden: Springer.

Vollmer, L. (2021). Gemeinsam für eine andere Wohnungspolitik!Warum eine Kooperation von Mieter*innenvereinen und Mieter*innenbewegung notwendig ist. Comun, 3(5), 12.

Vollmer, L., \& Kadi, J. (2018). Postneoliberaler Paradigmenwechsel oder punktuelle Beruhigungspolitik? Wohnungspolitik in der Krise des Neoliberalismus in Berlin und Wien. Prokla. Zeitschrift für kritische Sozialwissenschaften, 48(191), 247-264.

Williams, S. (2013). Fordert Alles. Lehren aus dem Transformativen Organizing. New York: Rosa-Luxemburg-Stiftung. 